GA-A15885

UC-77

\title{
HOT HELIUM FLOW TEST FACILITY SUMMARY REPORT
}

\author{
by \\ PROJECT STAFF
}

\author{
Prepared under \\ Contract DE-AT03-76ET35300 \\ for the San Francisco Operations Office \\ Department of Energy
}

\section{DATE PUBLISHED: JUNE 1980}




\section{DISCLAIMER}

This report was prepared as an account of work sponsored by an agency of the United States Government. Neither the United States Government nor any agency Thereof, nor any of their employees, makes any warranty, express or implied, or assumes any legal liability or responsibility for the accuracy, completeness, or usefulness of any information, apparatus, product, or process disclosed, or represents that its use would not infringe privately owned rights. Reference herein to any specific commercial product, process, or service by trade name, trademark, manufacturer, or otherwise does not necessarily constitute or imply its endorsement, recommendation, or favoring by the United States Government or any agency thereof. The views and opinions of authors expressed herein do not necessarily state or reflect those of the United States Government or any agency thereof. 


\section{DISCLAIMER}

Portions of this document may be illegible in electronic image products. Images are produced from the best available original document. 
GA-A15885

UC-77

\title{
HOT HELIUM FLOW TEST FACILITY SUMMARY REPORT
}

\author{
by \\ PROJECT STAFF

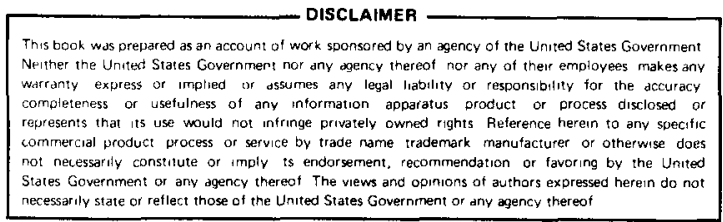 \\ Prepared under \\ Contract DE-AT03-76ET35300 \\ for the San Francisco Operations Office \\ Department of Energy
}

\section{GENERAL ATOMIC PROJECT 6400 DATE PUBLISHED: JUNE 1980}

Etsthibution of this gocument is undimited

\section{GENERAL ATOMIC COMPANY}




\section{ABSTRACT}

This report sumnarizes the results of a study conducted to assess the feasibility and cost of modifying an existing circulator test facility (CTF) at General Atomic Company (GA). The CTF originally was built to test the Delmarva Power and Light Co. steam-driven circulator. This circulator, as modified, could provide a source of hot, pressurized helium for hightemperature gas-cooled reactor (HTGR) and gas-cooled fast breeder reactor (GCFR) component testing. To achieve this purpose, a high-temperature impeller would be installed on the existing machine.

The projected range of tests which could be conducted for the project is also presented, along with corresponding cost considerations. 
CONTENTS

ABSTRACT . . . . . . . . . . . . . . . . . . . iii

1. Summary . . . . . . . . . . . . . . . . . . . 1

2. EXISTING CIRCULATOR TEST FACILITY . . . . . . . . . . . 2

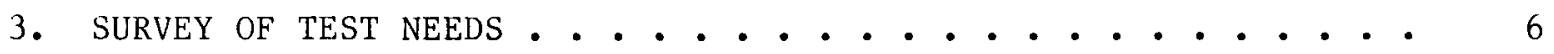

4. SELECTION OF DESIGN POINT FOR COMPRESSOR . . . . . . . . . 13

5. INSTALLATION OF CIRCULATOR . . . . . . . . . . . . . 17

5.1. Piping................ 17

5.2. Heat Exchanger . . . . . . . . . . . . . 18

6. USE OF FACILITY FOR GASES OTHER THAN HELIUM . • • • • • • • . 25

7. CONCLUSION . . . . . . . . . . . . . . . . 27

\section{FIGURES}

2-1. Overall view of circulator test facility . . . . . . . 3

2-2. Circulator test vessel .............. . . 4

2-3. Control panels for circulator test facility . . . . . . 5

4-1. HHFTF compressor performance, 8000-rpm design . . . . . . 15

4-2. HHFTF compressor design . . . . . . . . . . . . . 16

5-1. Circulator installation . . . . . . . . . . 19

5-2. Elevation view of HHFTF piping . . . . . . . . . . 20

5-3. Plan view of HHFTF piping . . . . . . . . . . . . 21

5-4. Typical cross section of helium piping, showing internal insulation . . . . . . . . . . . . . . . 22

5-5. Flange arrangement for HHFTF . . . . . . . . . . 23

5-6. Details of HHFTF heat exchanger . . . . . . . . . . 24

TABLES

3-1. Thermal barrier, reactor internals . . . . . . . . 8

3-2. Heat exchanger, HTGR, GCFR . . . . . . . . . . . 9

3-3. Control and Electrical . . . . . . . . . . . . 10 
TABLES (Continued)

3-4. Very high temperature reactor (VHTR) . . . . . . . . . 11

3-5. Other test programs . . . . . . . . . . . . . 12

4-1. Summary of HHFTF compressor design . . . . . . . . . . 13

6-1. Summary of costs to design, manufacture, and commission the HHFTF . . . . . . . . . . . . . . . 26 


\section{SUMMARY}

This report summarizes the results of a study conducted to assess the feasibility and cost of modifying an existing circulator test facility (CTF) at General Atomic Company (GA). The CTF originally was built to test the Delmarva Power and Light Co. steam-driven circulator. This circulator, as modified, could provide a source of hot, pressurized helium for hightemperature gas-cooled reactor (HTGR) and gas-cooled fast breeder reactor (GCFR) component testing. To achieve this purpose, a high-temperature impeller would be installed on the existing machine.

The projected range of tests which could be conducted for the project is also presented, along with corresponding cost considerations. 


\section{EXISTING CIRCULATOR TEST FACILITY}

The CTF at GA originally was constructed to test the prototype steam-turbine-driven helium circulators for the large HTGR and then eventually to perform production acceptance tests on all manufactured circulators. The facility (see Figs. 2-1 through 2-3), completed in 1974, consists primarily of the following:

1. An 8000-hp (5970 kW), motor-driven steam compressor. (This unit provides the steam required to drive the steam-turbine-driven circulator to $8000 \mathrm{rpm}$ with a power rating of $3000 \mathrm{HP}(2240 \mathrm{~kW})$.

2. A bearing water module. (This unit provides other services required of the circulator such as lubricant, buffer flow to the seals, and safe shutdown.)

3. All controls and instruments to operate the circulator and steam compressor.

During 1978 , a circulator originally intended for delivery to the Delmarva Power and Light Co. was tested in the facility. This previously tested source of helium flow can be made readily available for the hot helium flow test facility (HHFTF) simply by modifying the wheel on the Delmarva circulator and adding the high-temperature piping. 


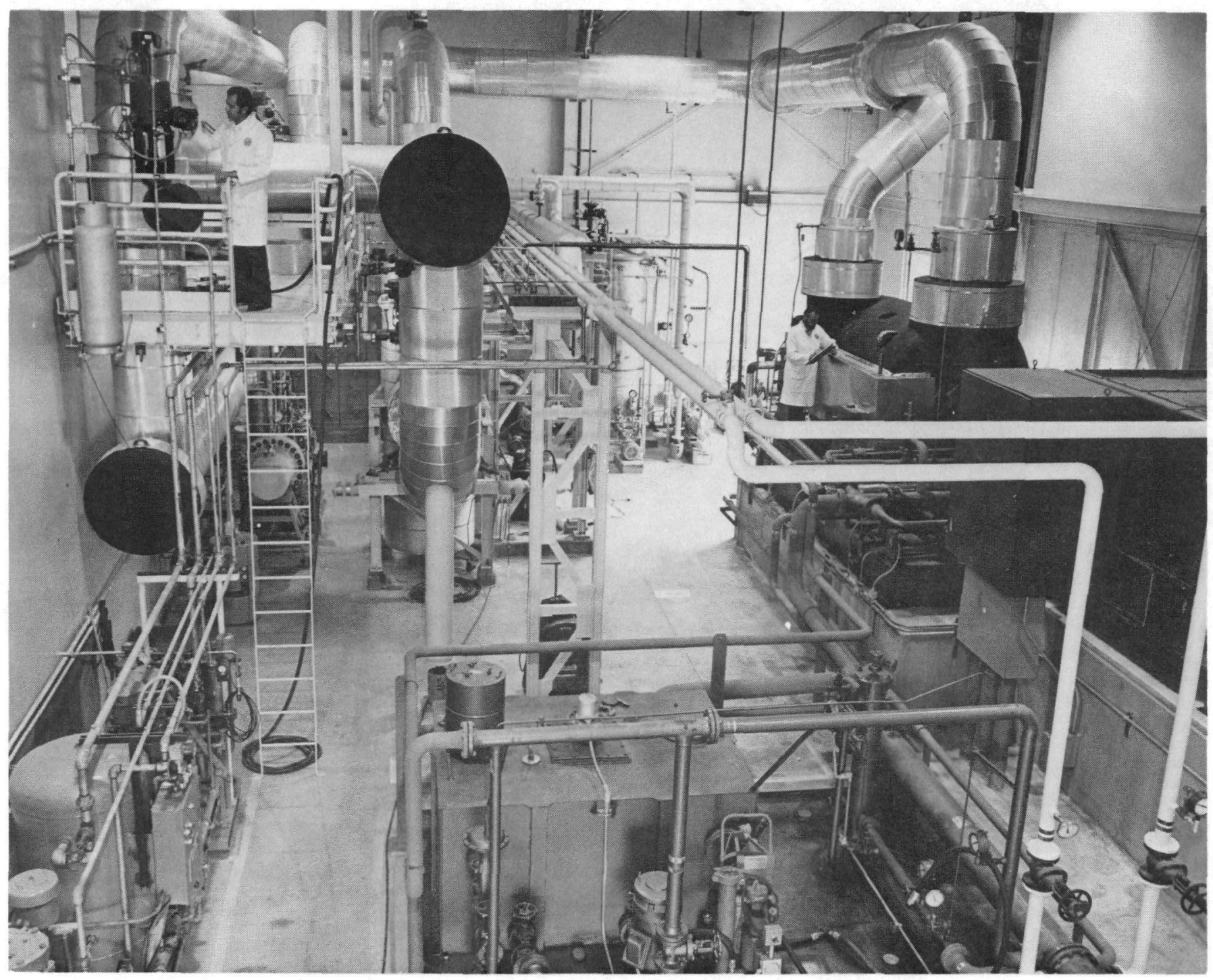

Fig. 2-1. Overall view of circulator test facility 


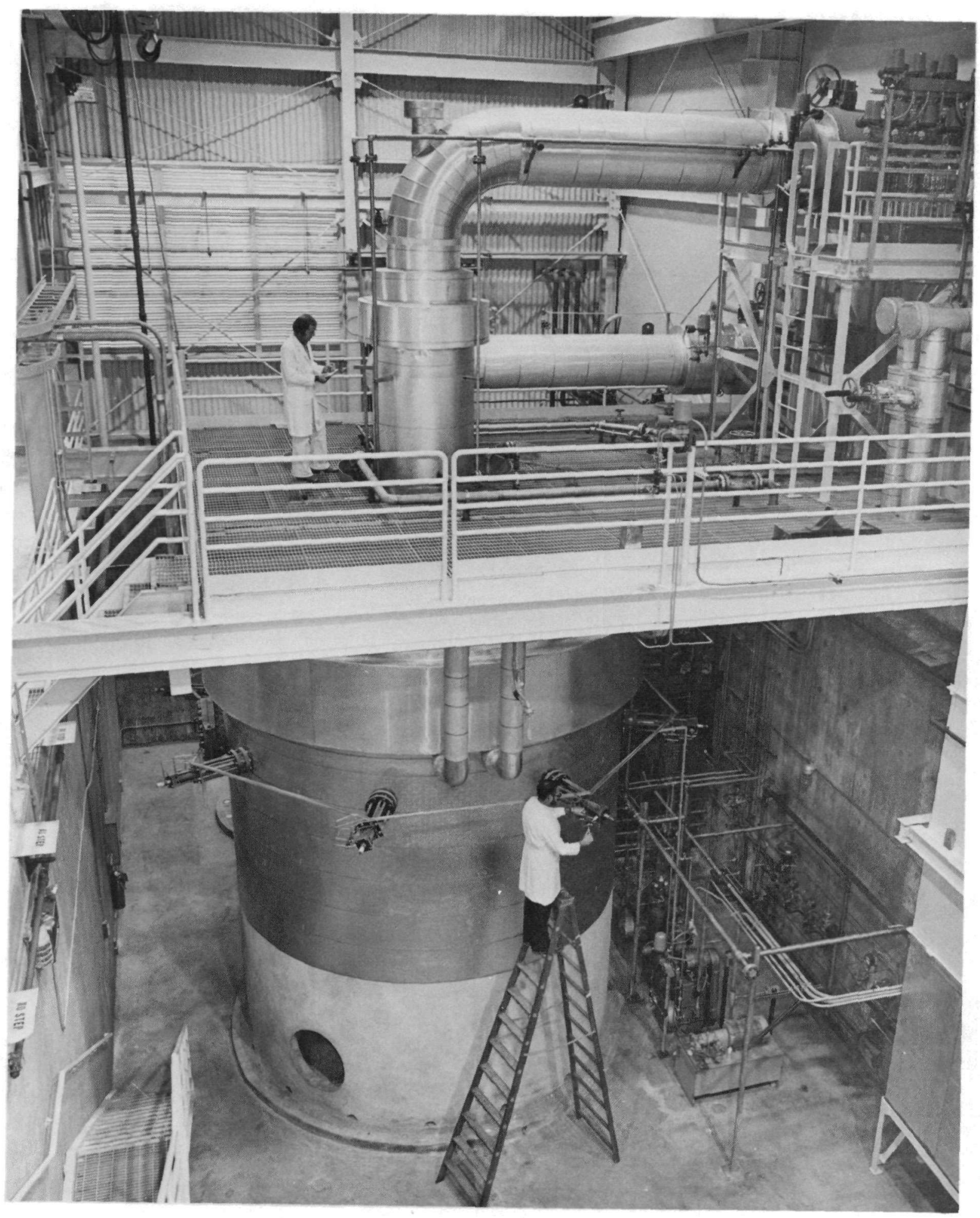

Fig. 2-2. Circulator test vesse1 


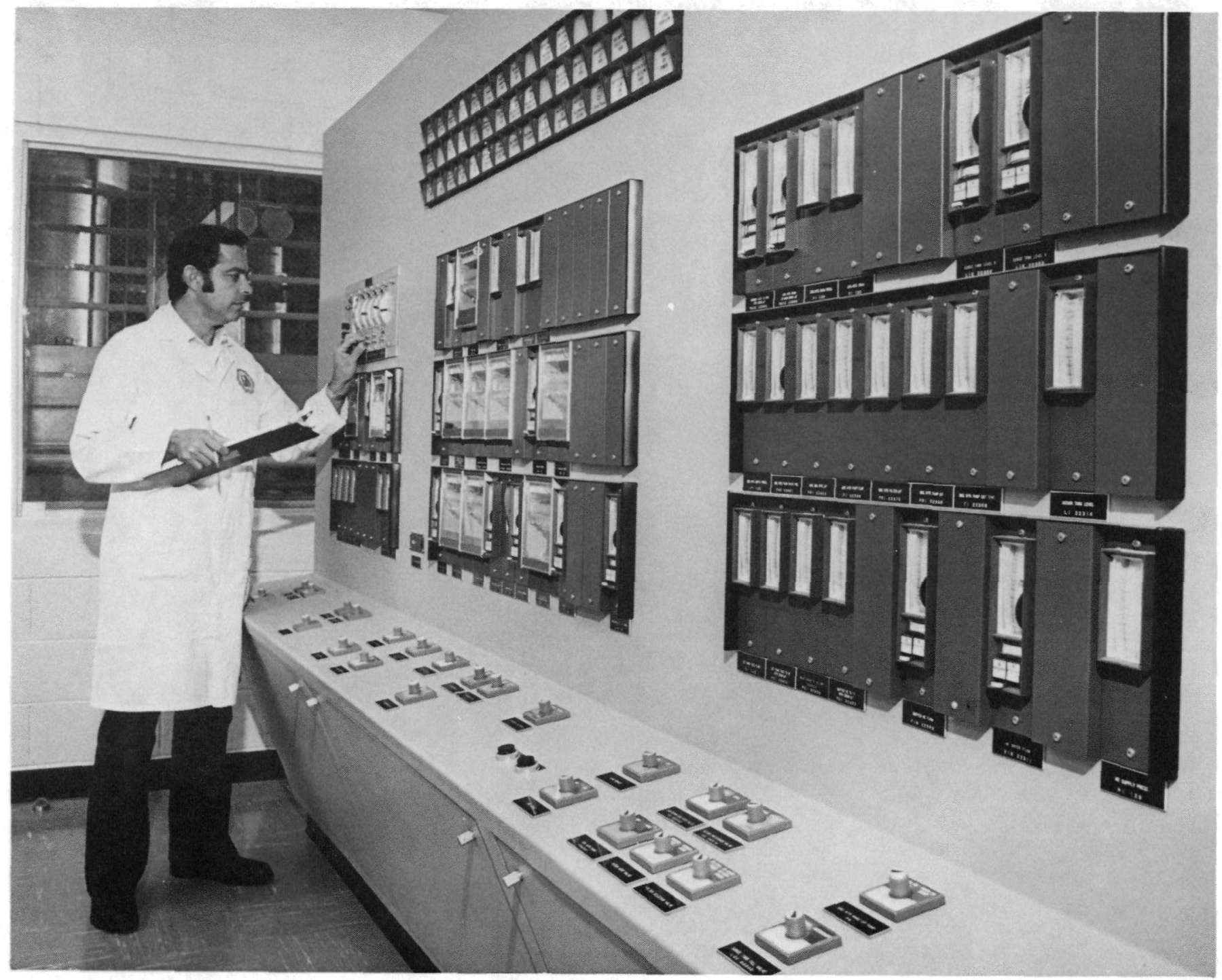

Fig. 2-3. Control panels for circulator test facility 


\section{SURVEY OF TEST NEEDS}

A survey of test needs, based on the needs of the HTGR and GCFR programs, was compiled prior to starting the preliminary design of HHFTF.

Table 3-1 summarizes the potential thermal barrier and reactor internals tests. These tests include helium flows to $38.6 \mathrm{~kg} / \mathrm{s}(85 \mathrm{lb} / \mathrm{s})$, temperatures to $954^{\circ} \mathrm{C}\left(1750^{\circ} \mathrm{F}\right)$, and pressures to $7170 \mathrm{kPa}$ (1040 psia) with negligible pressure drops. It is expected that all test specimens will fit easily into the facility.

Table 3-2 summarizes the heat exchange tests. These particular tests cover helium flows to $41 \mathrm{~kg} / \mathrm{s}(90 \mathrm{lb} / \mathrm{s})$, temperatures to $954^{\circ} \mathrm{C}\left(1750^{\circ} \mathrm{F}\right)$, and pressures to $8493 \mathrm{kPa}(1232 \mathrm{psia})$. The highest pressure drop, $69 \mathrm{kPa}$ (10 psia), occurs at a lower helium flow. Dimensionally, only the steam generator test presents a problem because of its $11.3-\mathrm{m}(37-\mathrm{ft}$ ) height. If the steam generator does not fit into the pit area, it could be installed outdoors with larger piping runs. As expected, however, all of the heat exchanger tests will require additional heat input to the loop beyond the 1.9 MW which the helium circulator can provide.

Table 3-3 covers the control and electrical tests. As noted from the table, all these tests can be run in conjunction with the steam generator or other tests.

The very high temperature reactor (VHTR) tests are summarized in Table 3-4. The flows and pressure drops are modest and only the helium-cooled core auxiliary heat exchanger (CAHE) requires a higher temperature $\left[1038^{\circ} \mathrm{C}\right.$ $\left.\left(1900^{\circ} \mathrm{F}\right)\right]$ than is needed for previous tests. The reformer definitely will have to be tested outdoors because of its height. In part, the reformer will require a new process gas loop to be designed and tested. Of course, 
the steam methane loop will not be considered a part of test facility capital expense. Additional heaters will be required for the CAHE test.

Al1 other component tests are grouped into Table 3-5. The most severe requirements are for the proposed GCFR core assembly prototype test. The $207 \mathrm{kPa}$ (30 psia) and $11,040 \mathrm{kPa}(1600 \mathrm{psia})$ pressure drops are both higher than for any other proposed test. 
TABLE $3-1$
THERMAL BARRIER, REACTOR INTERNALS

\begin{tabular}{|c|c|c|c|c|c|c|c|c|}
\hline & $\begin{array}{l}\text { Program } \\
\text { Department } \\
\text { Component }\end{array}$ & $\begin{array}{c}W_{\max } \\
{[\mathrm{kg} / \mathrm{s}(1 \mathrm{~b} / \mathrm{s})]}\end{array}$ & {$\left[{ }^{\circ} \mathrm{C}\left(\begin{array}{c}\max \\
\left({ }^{8}\right.\end{array}\right.\right.$} & $\begin{array}{r}\mathrm{P}_{\max } \\
{[\mathrm{kPa}(\mathrm{psia})]} \\
\end{array}$ & $\begin{array}{c}\Delta P_{\max } \\
{[\mathrm{kPa}(\mathrm{psi})]}\end{array}$ & $\begin{array}{l}\text { Geometric Dimensions } \\
\text { of Test Specimen } \\
{[\mathrm{m}(\mathrm{ft})]}\end{array}$ & Test ob jectives & Corments \\
\hline & Hot duct cest & $38.6(85)$ & $\begin{array}{l}838(1540)(\mathrm{SC})(\mathrm{a}) \\
954(1750)(\mathrm{GT})(\mathrm{a}) \\
510(950)(\mathrm{GCFR})\end{array}$ & $\begin{array}{l}5033(730)(\mathrm{SC}, \mathrm{GT}) \\
7170(1040)(\mathrm{GCFR})\end{array}$ & $3.4(0.5)$ & $\begin{array}{l}\text { I.D: } 1.52(5) \\
\text { Length: } 3.05(10) \\
\text { Liner diameter: } 2.13(7)\end{array}$ & $\begin{array}{l}\text { Verify thermal and } \\
\text { structural perfor- } \\
\text { mance of hot duct } \\
\text { thermal barrier in } \\
\text { realistic operating } \\
\text { environment }\end{array}$ & $\begin{array}{l}\text { Flow rate based on } \\
100.6 \text { mps }(330 \mathrm{fps}) \\
\text { annulus. Full duct } \\
\text { flow is } 408.2 \mathrm{~kg} / \mathrm{s} \\
(9001 \mathrm{~b} / \mathrm{s})\end{array}$ \\
\hline & $\begin{array}{l}\text { Class } C \text { thermal } \\
\text { barrier perfor- } \\
\text { mance test }\end{array}$ & $38.6(85)$ & $\begin{array}{l}838(1540)(\mathrm{SC})(\mathrm{a}) \\
954(1750)(\mathrm{GT})(\mathrm{a})\end{array}$ & $5033(730)(\mathrm{SC})$ & $1.4(0.2)$ & $\begin{array}{l}2.44 \times 2.44 \times 0.46 \\
(8 \times 8 \times 1.5) \\
\text { Ful1-scale class C model } \\
\text { [flow area to g1ve } 15.24 \\
\text { mps }(50 \mathrm{fps}) \mathrm{gas} \\
\text { velocity] }\end{array}$ & $\begin{array}{l}\text { Verify thermal per- } \\
\text { formance of class C } \\
\text { thermal barrier in } \\
\text { flowing hellum } \\
\text { environment }\end{array}$ & \\
\hline 3. & $\begin{array}{l}\text { Class } A \text { and } B \\
\text { thermal barrier } \\
\text { performance } \\
\text { tests }\end{array}$ & $38.6(85)$ & $\begin{array}{l}\text { Class A: } \quad 538(1000)(a) \\
\text { Class B: }\end{array}$ & $\begin{array}{l}5033(730)(\text { (SC) } \\
7170(1040)(\mathrm{GCFR})\end{array}$ & $1.4(0.2)$ & $\begin{array}{l}2.44 \times 2.44(8 \times 8) \\
\text { Full-scale class A and } \\
\text { B thernal barrier mode1s } \\
\text { (same as above) }\end{array}$ & $\begin{array}{l}\text { Verlfy thermal per- } \\
\text { formance of class } A \\
\text { and B thermal barrier } \\
\text { in flowing environ- } \\
\text { ment. Verify thermal } \\
\text { barrier 1ntegrity fn } \\
\text { acoustic and flow- } \\
\text { Induced vibration } \\
\text { environment }\end{array}$ & $\begin{array}{l}\text { Noise leve1 } 155 \mathrm{~dB} \\
\text { (SC), } 165 \mathrm{~dB}+(\mathrm{GT})\end{array}$ \\
\hline 4. & Core support & $18.6(41)$ & $871(1600)$ & $5067(735)$ & Neg1igible & Size for 1 region for GT & Demonstration tests & \\
\hline
\end{tabular}

(a) Fmergency condition [normal condition temperatures approximately $760^{\circ} \mathrm{C}\left(1400^{\circ} \mathrm{F}\right)$ for SC and $871^{\circ} \mathrm{C}\left(1600^{\circ} \mathrm{F}\right)$ for $\mathrm{GT}$ ]. 
TABLE $3-2$
HEAT EXCHANGER, HTGR, GCFR

\begin{tabular}{|c|c|c|c|c|c|c|c|c|}
\hline & $\begin{array}{l}\text { Program } \\
\text { Department } \\
\text { Component }\end{array}$ & {$[(\mathrm{kg} / \mathrm{s})(1 \mathrm{~b} / \mathrm{s})]$} & {$\left[\left(\left(^{\left.\mathrm{T}_{\mathrm{max}} \mathrm{C}\right)}{ }^{\circ} \mathrm{\circ}\right)\right]\right.$} & {$\left[\mathrm{kPa} \underset{(\mathrm{p} \dot{\max }}{\left.\left.\mathrm{P}_{\mathrm{a}}\right)\right]}\right.$} & $\underset{[\mathrm{kPa}(\mathrm{PsI})]}{\Delta P_{\max }}$ & $\begin{array}{l}\text { Geometric Dimensions } \\
\text { of Test Specimen } \\
{[\text { [i (ft)] }}\end{array}$ & Test objectives & Comments \\
\hline 1. & Steam generator & $5.8(12.7)$ & $675(1248)$ & $4908(712)$ & $31(4.5)$ & $\begin{array}{l}\text { Diameter: } 2.74(9) \\
\text { Helght: } 11.28(37) \\
\text { (overall dimensions) }\end{array}$ & Steatil generator performance & $3.5 \mathrm{MW}(\mathrm{t})$ heat \\
\hline 2. & Helical caHe & $\begin{array}{l}4.5(10) \\
\text { (see "Coments" } \\
\text { column) }\end{array}$ & $648(1200)$ & $8493(1232)$ & $3.4(0.5)$ & $\begin{array}{l}\text { Deameter: } 2.13(7) \\
\text { Height: } 4.57(15)\end{array}$ & CAHE performance (helical) & $\begin{array}{l}\text { Actual flow depending on model size } \\
\text { ( } 3 \text { MW }) \text { heat }\end{array}$ \\
\hline 3. & Bayonet CAHE & $\begin{array}{l}40.8(90) \\
\text { (see "Comments" } \\
\text { column) }\end{array}$ & $954(1750)$ & $7583(1100)$ & $6.9(1.0)$ & $\begin{array}{l}\text { Diameter: } 1.83(6) \\
\text { Height: } 4.27(14)\end{array}$ & Bayonet bundle performance & $\begin{array}{l}105 \mathrm{~mW} \text { heat full-scale complete } \\
\text { section mode1 (could be a full- } \\
\text { scale } 1 / 2 \text { section model for less flow) }\end{array}$ \\
\hline 4. & $\begin{array}{l}\text { Finned tube heat } \\
\text { transfer and } \\
\text { pressure drop }\end{array}$ & $12.2(27)$ & $223(433)$ & $3206(465)$ & $69(10)$ & $0.002^{2}\left(2^{2}\right) \times 0.61$ (2) 1ong & $\begin{array}{l}\text { Short fin tube heat transfer } \\
\text { and pressure drop precooler } \\
\text { for GT application }\end{array}$ & $\begin{array}{l}\text { Heat transfer rate depends on tube } \\
\text { side conditions. Not critical }\end{array}$ \\
\hline 5. & $\begin{array}{l}\text { Insulation and } \\
\text { thermal drop }\end{array}$ & & $954(1750)$ & & & Relatively small & [stablish detall design & $\begin{array}{l}\text { To be defined later in the } \\
\text { preliminary design phase }\end{array}$ \\
\hline
\end{tabular}


TABLE 3-3

CONTROL AND ELECTRICAL

\begin{tabular}{|c|c|c|c|c|c|c|c|}
\hline $\begin{array}{c}\text { Program } \\
\text { Compartment } \\
\text { Component }\end{array}$ & $\begin{array}{c}W_{\max } \\
{[\mathrm{kg} / \mathrm{s}(1 \mathrm{~b} / \mathrm{s})]}\end{array}$ & {$\left[0^{\circ} \mathrm{C}\left({ }^{\max } \mathrm{F}\right)\right]$} & $\begin{array}{c}\mathrm{P}_{\max } \\
{[\mathrm{kPa}(\mathrm{ps} 1 \mathrm{a})]}\end{array}$ & $\begin{array}{c}\Delta \mathrm{P}_{\max } \\
{[\mathrm{kPa}(\mathrm{psi})]}\end{array}$ & $\begin{array}{c}\text { Geometric Dimensions } \\
\text { of Test Specimens }\end{array}$ & Test Object Ives & Comments \\
\hline $\begin{array}{l}\text { 1. Steam generator inlet } \\
\text { temperature rake } \\
\text { measurement system }\end{array}$ & (a) & (a) & (a) & $3.4(0.5)$ & Full scale & $\begin{array}{l}\text { 1. Flow induced vibration } \\
\text { 2. Sensor time constant }\end{array}$ & \\
\hline $\begin{array}{l}\text { 2. Moisture monitor } \\
\text { qualification }\end{array}$ & (a) & $343(650)$ & $\begin{array}{l}5170(750) \mathrm{GT} \\
7239(1050) \mathrm{SC}\end{array}$ & Negligible & Fu11 scale & $\begin{array}{l}\text { 1. Rake design } \\
\text { 2. Response } \\
\text { 3. Heat tracing } \\
\text { 4. Flow balance }\end{array}$ & \\
\hline 3. Helium flow & (a) & $343(650)$ & $\begin{array}{l}5170(750) \mathrm{GT} \\
7239(1050) \mathrm{SC}\end{array}$ & $34(5.0)$ & & Flow calibration & \\
\hline
\end{tabular}

(a) Run in conjunction with steam generator tests. See Table $3-2$, itell no. 1 . 
TABLE 3-4

VERY HIGH TEMPERATURE REACTOR (VHTR)

\begin{tabular}{|c|c|c|c|c|c|c|c|c|}
\hline & $\begin{array}{l}\text { Program } \\
\text { Department } \\
\text { Component }\end{array}$ & $\frac{W_{\max }}{[\mathrm{kg} / \mathrm{s}(1 \mathrm{~b} / \mathrm{s})]}$ & {$\left[{ }^{\circ} C^{T_{\max }}\left({ }^{\circ} F\right)\right]$} & 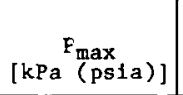 & 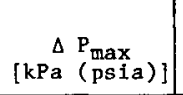 & $\begin{array}{l}\text { Geometric Dimensions } \\
\text { of Test Specimens }\end{array}$ & Test objectives & Corments \\
\hline 1. & $\begin{array}{l}\text { Intermediate heat } \\
\text { exchanger module }\end{array}$ & $\begin{array}{l}0.68(1.5) \\
\text { per IHX module }\end{array}$ & $\begin{array}{l}815-985 \\
(1500-1742)\end{array}$ & $4998(725)$ & $55-69(8-10)$ & $\begin{array}{l}\text { Module is hexagonal } \\
. .18 \mathrm{~m}(7 \mathrm{n} \text {.) } \\
\text { across flats by } 9.7 \mathrm{~m} \\
\text { ( } 32 \mathrm{ft} \text { ) long. (Test } \\
\text { in a vertical position) }\end{array}$ & $\begin{array}{l}\text { Heat transfer per- } \\
\text { formance and pres- } \\
\text { sure drop character- } \\
\text { istics for various } \\
\text { enhanced surfaces }\end{array}$ & $\begin{array}{l}1.6 \mathrm{MW}(t) \text { heat } \\
\text { duty per module. } \\
\text { Multiple modules } \\
\text { would be tested }\end{array}$ \\
\hline 2. & Reformer & $\begin{array}{l}0.14(0.3) \\
\text { per tube }\end{array}$ & $\begin{array}{l}649-899 \\
(1200-1650)\end{array}$ & $\begin{array}{l}5101(740) \\
\text { (shel1) }\end{array}$ & $\begin{array}{l}69(12) \\
\text { (she11) }\end{array}$ & $\begin{array}{l}0.09 \mathrm{~m}(3.5 \mathrm{in}) \times 14.3 \\
\mathrm{~m}(47 \mathrm{ft}) \text { tube. (Test } \\
\text { in a vertical position) }\end{array}$ & $\begin{array}{l}\text { Develop process } \\
\text { kinetic behavior for } \\
\text { steam methane re- } \\
\text { forming with this } \\
\text { nonconventional re- } \\
\text { former. Different } \\
\text { catalytic geometries } \\
\text { would be tested }\end{array}$ & $\begin{array}{l}\text { Process gas }\left(\mathrm{CH}_{4} \text {, }\right. \\
\left.\mathrm{H}_{2} \mathrm{O}, \mathrm{CO}, \mathrm{H}_{2}\right) \text { flows } \\
\text { fnside reformer } \\
\text { tubes at } 0.08 \mathrm{~kg} / \mathrm{s} \\
(0.171 \mathrm{~b} / \mathrm{s}) \mathrm{per} \\
\text { tube. } 170 \mathrm{KW}(\mathrm{t}) \\
\text { heat duty per tube }\end{array}$ \\
\hline 3. & Thermal barrier & $\begin{array}{l}\text { Modest at } \\
122 \text { mps ( } 400 \mathrm{fps})\end{array}$ & $985(1742)$ & $4998(725)$ & & $\begin{array}{l}\text { Representative } 0.62 \mathrm{~m} \\
(2 \mathrm{ft}) \times 0.62 \mathrm{~m}(2 \mathrm{ft}) \\
\text { panels }\end{array}$ & $\begin{array}{l}\text { Vibration, permea- } \\
\text { tion, pressure drop } \\
\text { erosion }\end{array}$ & \\
\hline 4. & Helfum-cooled CAHE & $\begin{array}{l}2.27-3.2(5-7) \\
\text { for fuil sized } \\
\text { model }\end{array}$ & $\begin{array}{l}927-1038 \\
(1700-1900)\end{array}$ & $\begin{array}{l}172(25) \\
\text { depress; } \\
4998(725) \\
\text { press }\end{array}$ & $14-34(2-5)$ & $\begin{array}{l}\text { Straight tube - bayonet } \\
\text { tube }\end{array}$ & $\begin{array}{l}\text { Establish perfor- } \\
\text { mance data and mech- } \\
\text { anical/aerodynamic } \\
\text { response. Pres- } \\
\text { surized and unpres- } \\
\text { surized shell side - } \\
\text { low flow stability } \\
\text { tests }\end{array}$ & $\begin{array}{l}\text { Full size model } \\
\text { need not be tested. }\end{array}$ \\
\hline & Hot duct & & & & & & & $\begin{array}{l}\text { See Table 3-1, } \\
\text { 1tem No. } 1\end{array}$ \\
\hline
\end{tabular}


TABLE 3-5

OTHER TEST PROGRAMS

\begin{tabular}{|c|c|c|c|c|c|c|c|c|}
\hline & $\begin{array}{l}\text { Program } \\
\text { Department } \\
\text { Component }\end{array}$ & $\underset{[\mathrm{kg} / \mathrm{s}(1 \mathrm{~m} / \mathrm{s})]}{W_{\max }}$ & {$\left[{ }^{\circ} \mathrm{C}\left({ }^{\mathrm{T}}{ }^{\mathrm{max}} \mathrm{F}\right)\right]$} & {$[\mathrm{kPa}(\mathrm{psia})]$} & $\underset{[\mathrm{kPa}}{\Delta \mathrm{p}_{\max }}$ & $\begin{array}{c}\text { Geometric Dimensions } \\
\text { of Test Specimens }\end{array}$ & Test Obfectives & Comments \\
\hline & $\begin{array}{l}\text { GCFR fuel element } \\
\text { assemblies }\end{array}$ & 6.8 (15) & $204(400)$ & $6894(1000)$ & $207(3)$ & $\begin{array}{l}0.20 \mathrm{~m}(8 \mathrm{in}) \text { hex } x \\
4.9 \mathrm{~m}(16 \mathrm{ft}) \text { long }\end{array}$ & $\begin{array}{l}\text { Flow-induced vibration } \\
\text { tests }\end{array}$ & $\begin{array}{l}\text { Up and down flows } \\
\text { would be desirable }\end{array}$ \\
\hline & Core graphite & (Later) & (Later) & (Later) & (Later) & (Later) & $\begin{array}{l}\text { 1. } \\
\text { 2. Lifitation } \\
\text { depressurization }\end{array}$ & \\
\hline 3. & $\begin{array}{l}\text { Natural convection } \\
\text { in the hot cross } \\
\text { duct for both GCFR } \\
\text { and HTGR }\end{array}$ & Not important & $538(1000)$ & $\begin{array}{l}12409(1800) \\
\text { desired; } 700 \\
\text { acceptable }\end{array}$ & Not important & $\begin{array}{l}\text { Dust approximately } \\
1.8 \mathrm{~m}(6 \mathrm{ft}) \text { diame- } \\
\text { ter } \mathrm{x} .6 \mathrm{~m}(15 \mathrm{ft}) \\
\text { long plus space for } \\
\text { an } \mathrm{Hx} \text {, approximately } \\
1.5 \mathrm{~m} \times 1.5 \mathrm{~m}(5 \mathrm{ft} \\
\times 5 \mathrm{ft})\end{array}$ & $\begin{array}{l}\text { 1. Determine heat } \\
\text { 1osses to CACS } \\
\text { 2. } \\
\text { Determine natural } \\
\text { convection veloci- } \\
\text { ties }\end{array}$ & $\begin{array}{l}\text { 1. Requires approx- } \\
\text { Imately ful1- } \\
\text { scale model } \\
\text { 2. Heat sink for } \\
\text { CACS model can } \\
\text { be combined with } \\
\text { the HX loop }\end{array}$ \\
\hline 4. & $\begin{array}{l}\text { Functional test of } \\
\text { helium isolation } \\
\text { valves }\end{array}$ & Not important & $371(700)$ & $\begin{array}{l}4825(700) \\
\text { acceptable }\end{array}$ & $\begin{array}{l}\text { Up to } 276 \\
(40) \text { ps1 }\end{array}$ & $\begin{array}{l}\text { Values up to } 0.91 \mathrm{~m} \\
(3 \mathrm{ft}) \text { diameter }\end{array}$ & $\begin{array}{l}\text { Confirm operational } \\
\text { characteristics of } \\
\text { values under true con- } \\
\text { ditions }\end{array}$ & \\
\hline & $\begin{array}{l}\text { LHTGR lower } \\
\text { plenum }\end{array}$ & $19(41)$ & $677(1250)$ & $5170(750)$ & $41(6.0)$ & $\begin{array}{l}\text { (See "Comments" } \\
\text { column) }\end{array}$ & $\begin{array}{l}\text { Study characteristics } \\
\text { of the hot streak mfx- } \\
\text { ing in the lower ple- } \\
\text { num }\end{array}$ & $\begin{array}{l}\text { One region, or } 16 \\
\text { regions at } 1 / 4 \text {-scale }\end{array}$ \\
\hline 6. & $\begin{array}{l}\text { LHTGR orifice con- } \\
\text { trol valve }\end{array}$ & $19(41)$ & $677(1250)$ & $5170(750)$ & $27(4.0)$ & Ful1-scale & $\begin{array}{l}\text { Determine loss co- } \\
\text { efficient for both } \\
\text { normal and reverse } \\
\text { flow }\end{array}$ & \\
\hline & Fusion blanket & $30(66)$ & $300(572)$ & $6080(882)$ & $69(10)$ & (Later) & $\begin{array}{l}\text { Flow tests, thermal } \\
\text { tests, pressure/tem- } \\
\text { perature cycling } \\
\text { tests, off-normal } \\
\text { operation tests }\end{array}$ & $\begin{array}{l}\text { Requires } 40 \mathrm{MW} \\
\text { heating }\end{array}$ \\
\hline
\end{tabular}




\section{SELECTION OF DESIGN POINT FOR COMPRESSOR}

The design point selected for the compressor is summarized in Table 4-1. This point was selected as a compromise to cover most of the proposed tests.

TABLE $4-1$

SUMMARY OF HHFTF COMPRESSOR DESIGN

\begin{tabular}{l|l}
\hline \multicolumn{1}{c|}{ Fluid } & \multicolumn{1}{|c}{ Helium } \\
\hline Temperature & $871^{\circ} \mathrm{C}\left(1600^{\circ} \mathrm{F}\right)$ \\
Pressure & $5515 \mathrm{kPa}(800 \mathrm{psia})$ \\
Flow rate & $20.45 \mathrm{~kg} / \mathrm{s}(45 \mathrm{lb} / \mathrm{s})$ \\
Pressure rise & $21 \mathrm{psid}(145 \mathrm{kPa})$ \\
\hline
\end{tabular}

The $871^{\circ} \mathrm{C}\left(1600^{\circ} \mathrm{F}\right)$ temperature noted in Table $4-1$ was picked as the maximum within the currently known metallurgical limits of materials available for turbomachinery. This temperature also covers 22 of the 28 possible tests envisioned. Additionally, some of the hotter tests require electric heaters because of the required heat loads. Thus, higher temperatures could be reached outside of the compressor area.

The $5515 \mathrm{kPa}$ (800 psia) value was selected primarily as a cost saving factor. This value covers 19 of the tests planned and could include many of the remaining tests, if analysis shows that temperature and fluid velocities are more important than absolute pressure. If a pressure greater than 5515 $\mathrm{kPa}$ (800 psia) were to be selected, the circulator service system and its primary closure would have to be redesigned and rebuilt at substantial cost. Additionally, the circulator bearing housing would have to be reanalyzed to establish its pressure limits. Another expensive item to consider, if higher pressures are selected, is the air blast heat exchanger. The reason for this is that the walls would need to be much thicker to withstand 
the increased pressure. The present cost of the material (f $\$ 200,000$ ) could well exceed $\$ 500,000$, if the pressure were increased to $12,409 \mathrm{kPa}$ (1800 psia).

The pressure drop covers all tests except for the GCFR core assembly prototype test. However, because of the long-term test proposed (four continuous years), it would probably not be feasible to run the HHFTF. For example, it is estimated that electrical costs for the facility to power the 8000-hp motor for the steam compressor and peripheral motors will be $\mathrm{f} \$ 200,000$ per quarter. For four years, this indicates a cost of $\$ 3,200,000$ for electricity alone. Using a separate compressor rated at 550-hp would lower this cost to $\$ 210,000$ over the four years.

The flow selected yields a good compromise design. It is low enough to prevent any stall operation of the machine, but is large enough to allow for efficient operation at the high-flow, low-head rise operating points.

The compressor will meet the variety of operating conditions through its variable speed control combined with a set of orifice and bypass sections. Figure 4-1 shows the performance curve for the compressor. For low-head, high-flow operating points (as depicted by point $A$ in the figure), the compressor can either operate at this point at a lower speed or, by placing an orifice in series with the compressor, the compressor can operate at design speed (point $A^{\prime}$ ). For low flow tests with high resistance (point $B$ in Fig. 4-1) the circulator would operate in stall. However, by adding a flow bypass loop, the compressor can be made to operate at point $B^{\prime}$. Adding a bypass and an orifice in series would make the compressor operate at point $B "$. With this method of orificing and bypassing, a wide variety of tests can be run, and the compressor can still operate at close to its design point and heat input.

Figure 4-2 shows the compressor designed for the HHFTF. The wheel will be cast out of IN-100. The ducts leading into the compressor and the diffuser will all be constructed of Hastelloy $X$. 


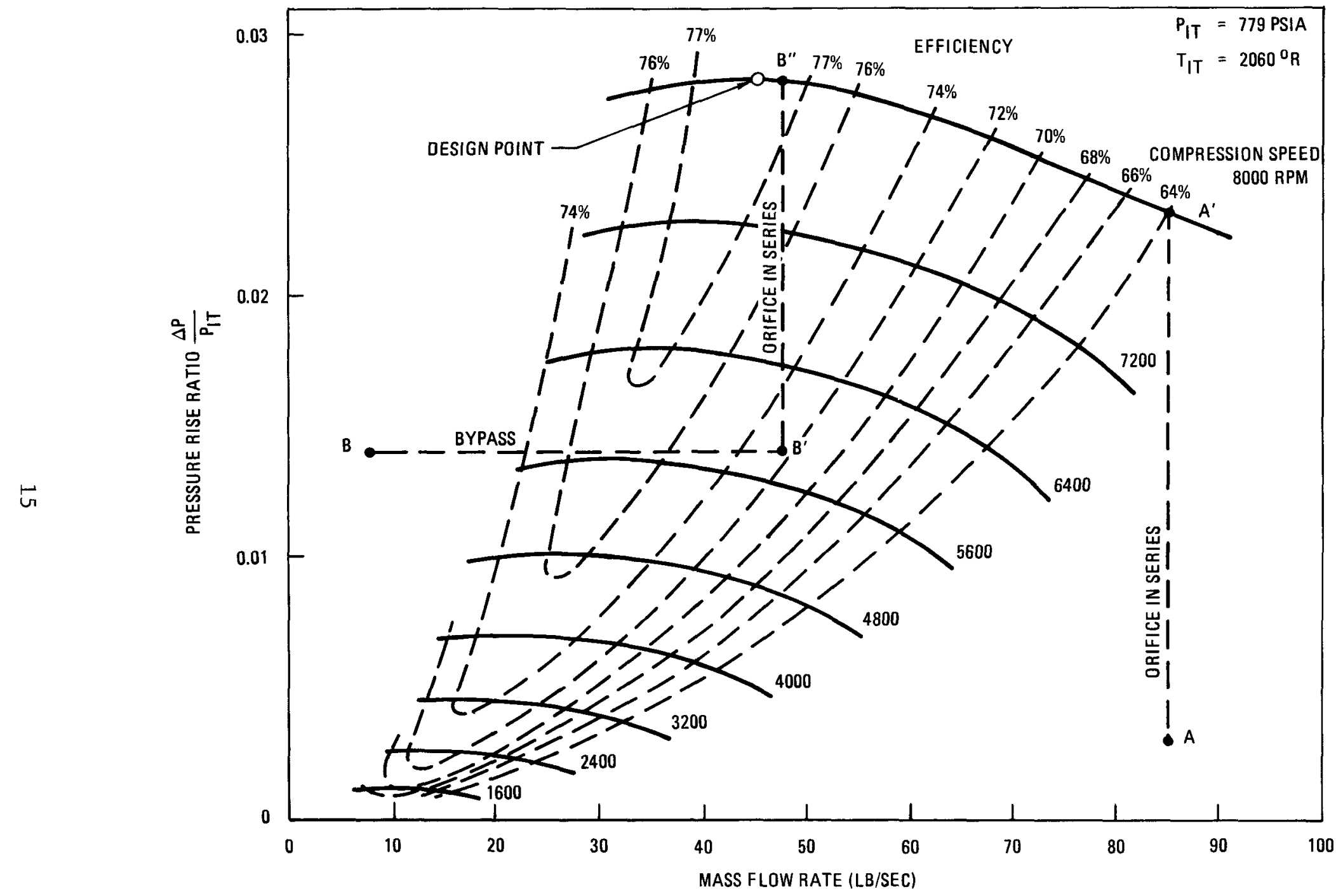

Fig. 4-1. HHFTF compressor performance, 8000-rpm design 


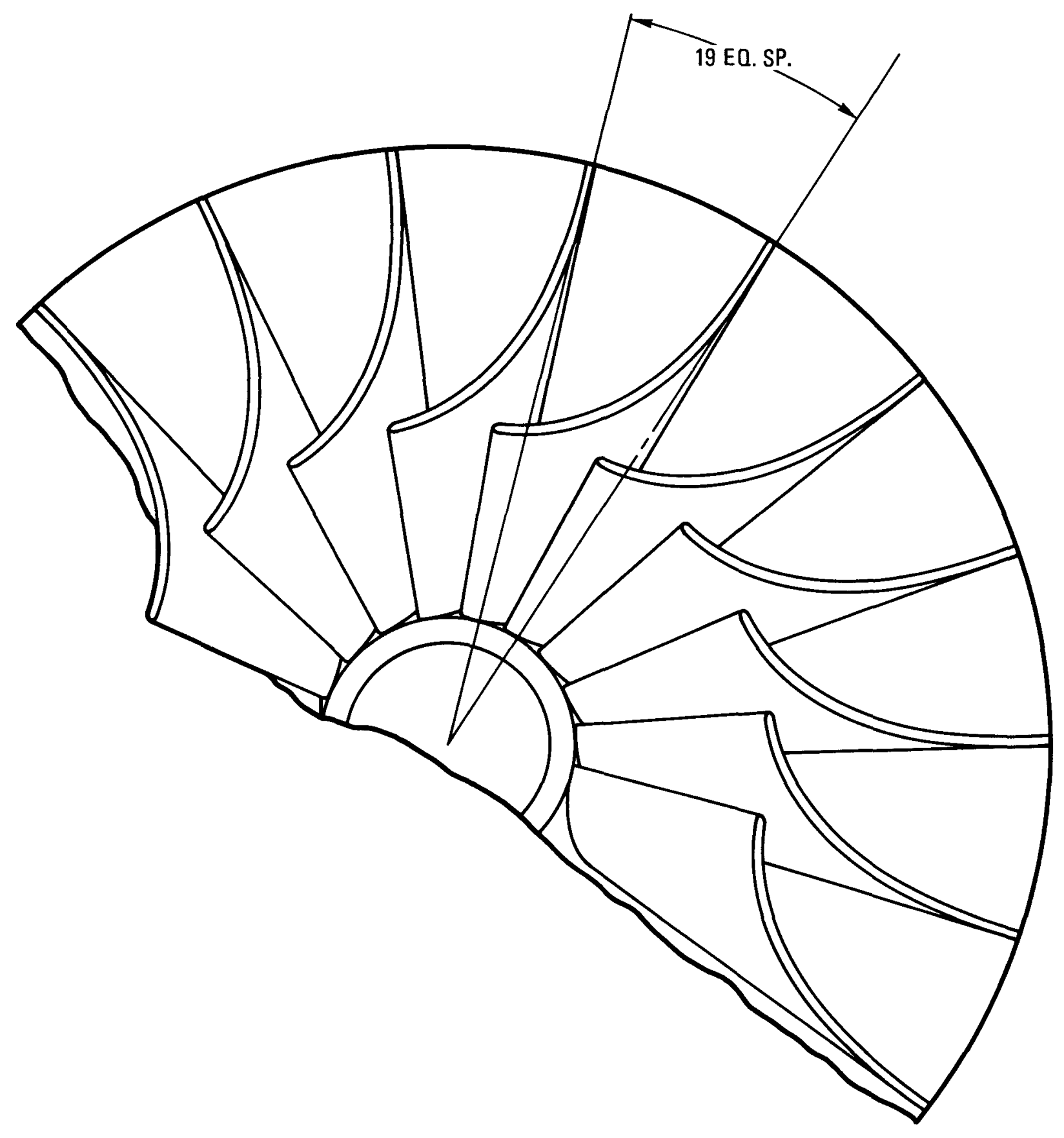

Fig. 4-2. HHFTF compressor design 


\section{INSTALLATION OF CIRCULATOR}

Figure 5-1 shows the installation of the circulator into the pressure housing. The main support for the housing is the existing vessel for the CTF. Since all service and steam lines to the circulator were based on the location of this existing vessel, it was deemed economically prudent to keep the vessel as a reference point. All internals of the vessel will have to be removed and holes cut in the bottom and sides. The modified circulator simply mounts on the original flange and forms part of the closure. Inside the vesse1 is a newly constructed pressure housing that mounts to the bottom of the original vesel flange. These three flanges, when bolted together, will form a closure for the helium. It should be noted that the internal insulation on the pressure housing also includes all of the added insulation around the circulator. The internal insulation is required to maintain the housing temperature below $371^{\circ} \mathrm{C}\left(700^{\circ} \mathrm{F}\right)$ so that carbon steel could be used instead of expensive high-temperature alloys. The added insulation on the circulator is required since it was designed for $316^{\circ} \mathrm{C}\left(600^{\circ} \mathrm{F}\right)$ helium on the outside of the original insulation.

\subsection{PIPING}

The circulator is only the source of helium flow and energy input. Piping is required to direct the helium from the circulator to a test area with more room. From the test area, the helium must then flow to and through a heat exchanger and then back to the compressor inlet. Figures 5-2, 5-3, and 5-4 show the piping arrangement selected. It should be noted that by arranging a pair of blind flanges, the test area can be made to easily accept upflow and downflow. This feature can be seen better on Fig. 5-5. If flanges $A$ and $C$ are blocked off, the helium flow is directed downward through flange $D$. Flange $B$ is up higher to accept the flow from the test area. If $f$ langes $B$ and $D$ are blocked off, flange $C$ directs the helium upward and flange $A$, close to the floor, can receive the flow from 
the fixture. The connector near flange $C$ is the bypass flange. An orifice in the flange controls the extent of bypass around the test fixture, allowing the circulator to run at full speed and to thereby maintain its intended power input.

Note that the piping also is insulated on the inside, allowing carbon steel to be used as the pressure-retaining boundary. The piping shown in Figs. 5-2 and 5-3 is what is considered part of the basic facility cost and is included in the cost estimate. Any additional piping going to the test fixture will have to be included in the cost of the particular test.

\subsection{HEAT EXCHANGER}

A heat exchanger is required in the test loop in order to control the helium temperature to the test fixture. The proposed design consists of a simple single "U" tube constructed out of Hastelloy $X$ with atmospheric air flowing over the outside of the tube to remove the excess heat. Because of the relatively low pressure vessel code allowable stress for Haste1loy $X$ at $871^{\circ} \mathrm{C}\left(1600^{\circ} \mathrm{F}\right)$, the heat exchanger also has to be insulated on the inner surface. This insulation consists of a thin sleeve of Hastelloy $X$ wrapped with $3.2 \mathrm{~mm}$ (1/8 in.) Hastelloy $X$ wire. This provides a thin pocket of stagnant helium gas, thereby reducing the temperature or the pressureretaining Hastelloy $X$ pipe and allowing a much higher stress to be used. See Fig. 5-6 for details on the exchanger.

The air flow to the exchanger is controlled with two $60 \%$ blowers in paralle1. Each blower will have a bypass system. The exhaust will pass through a silencer or reduce the sound pressure level to within the industry allowable. 




Fig. 5-1. Circulator installation 


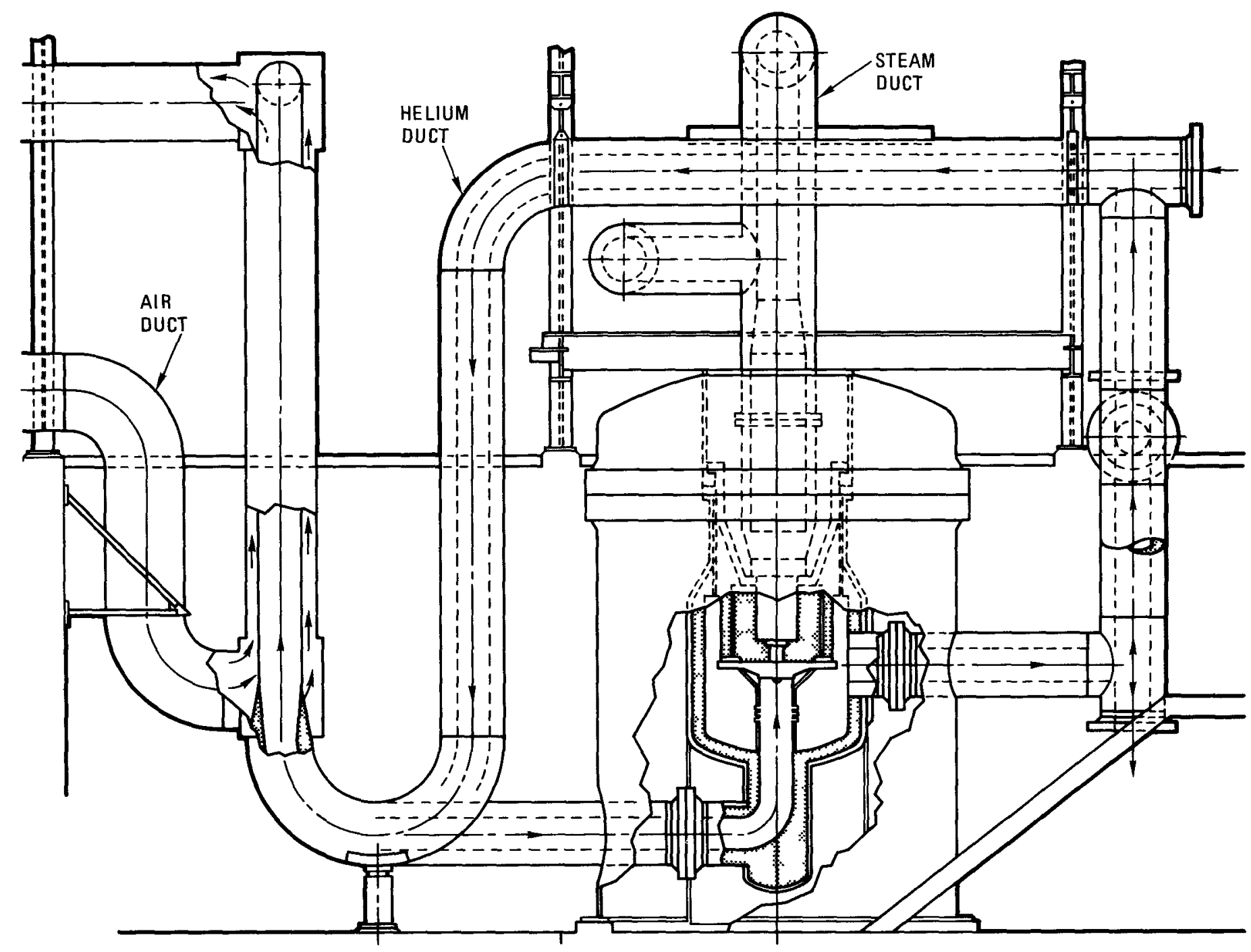

Fig. 5-2. Elevation view of HHFTF piping 


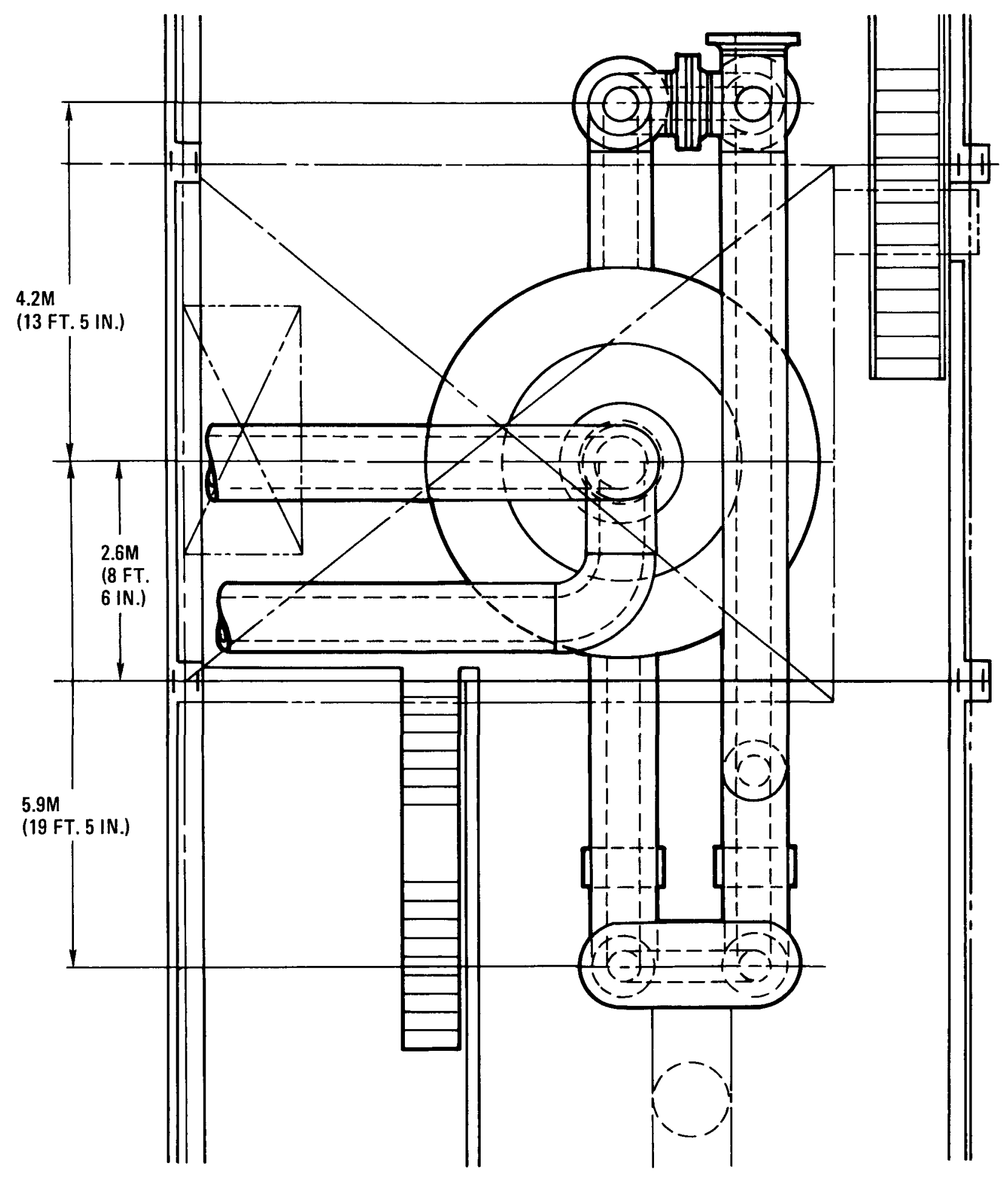

Fig. 5-3. Plan view of HHFTF piping 


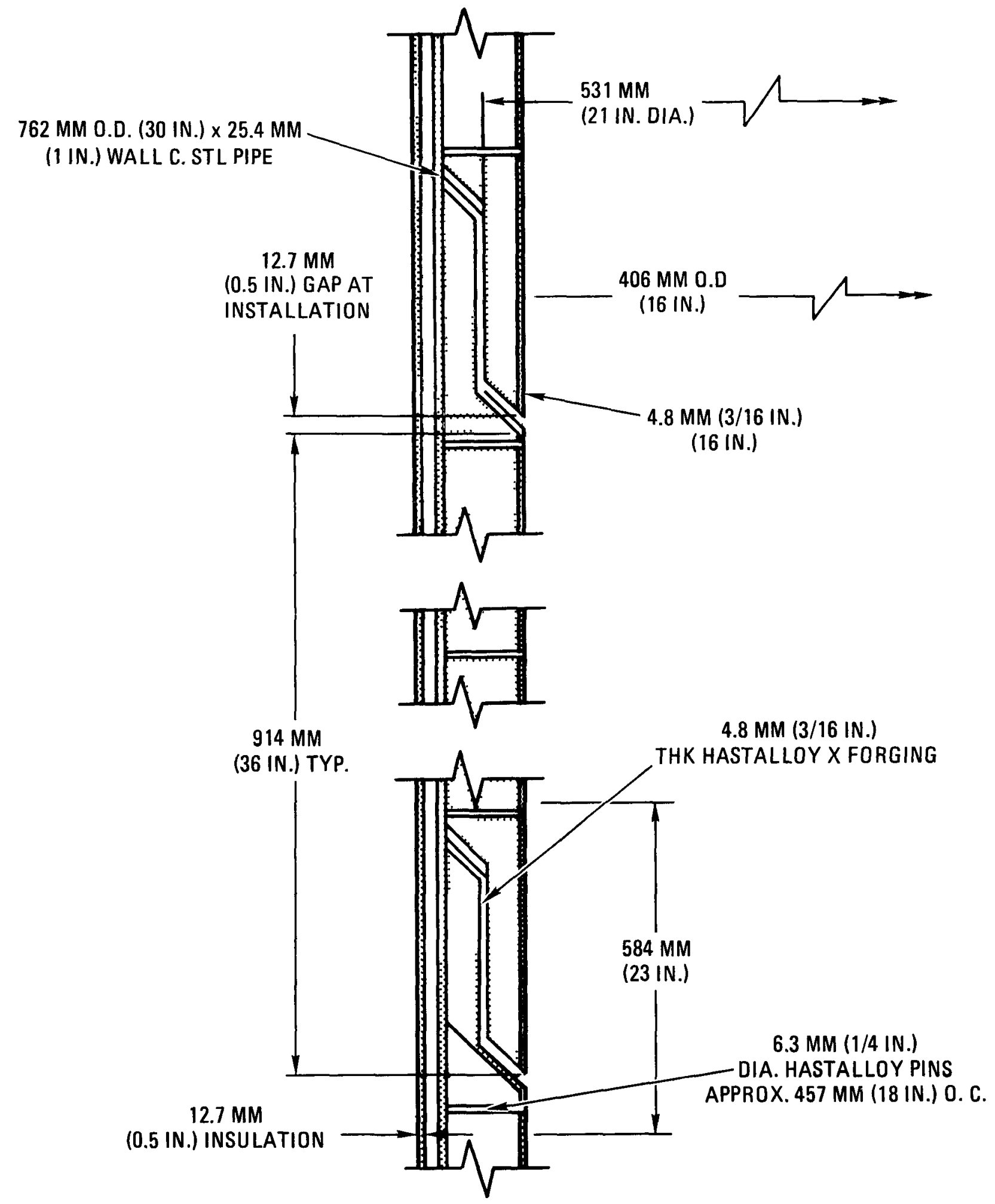

Fig. 5-4. Typical cross section of helium piping, showing internal insulation 


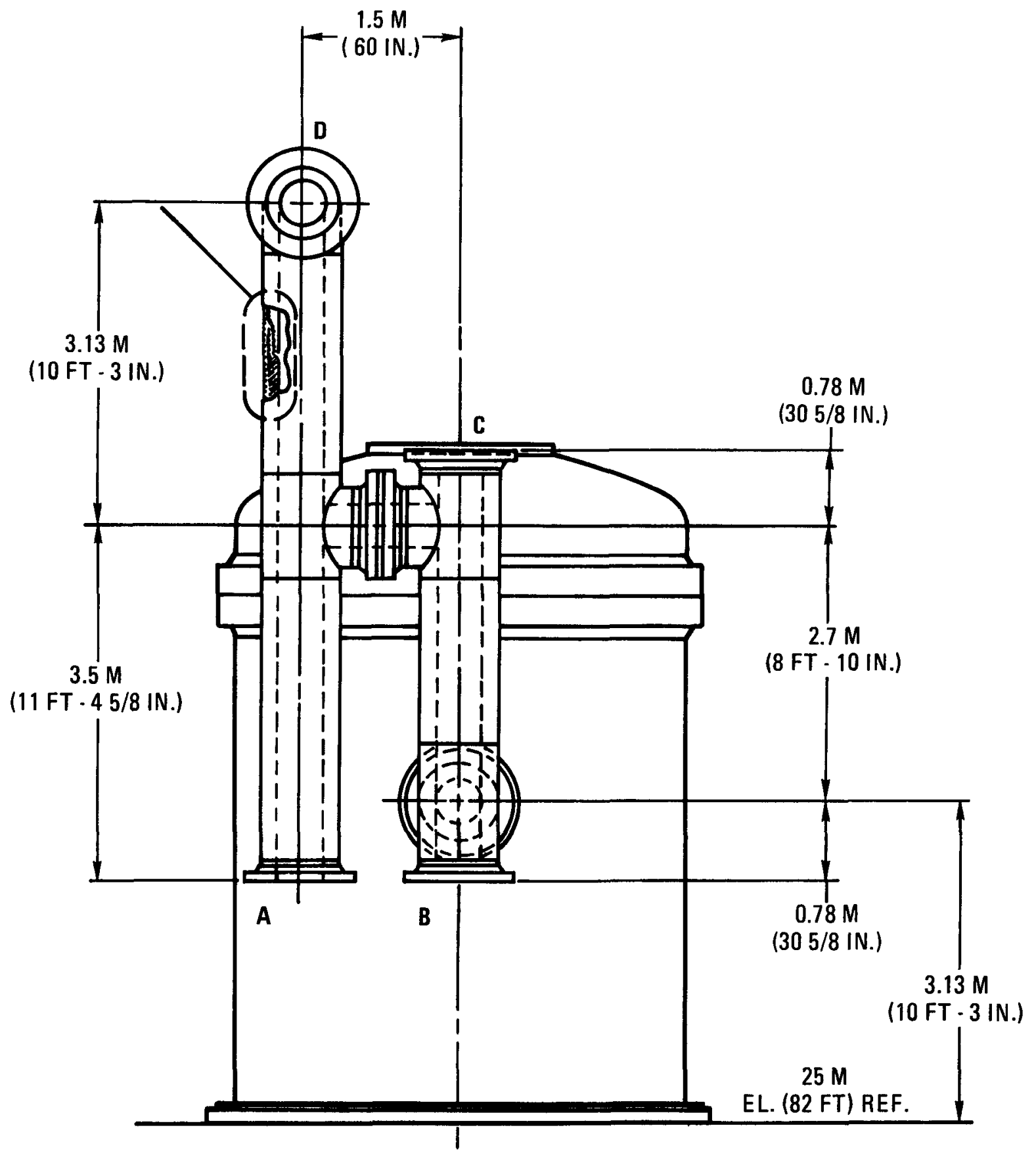

Fig. 5-5. Flange arrangement for HHFTF 


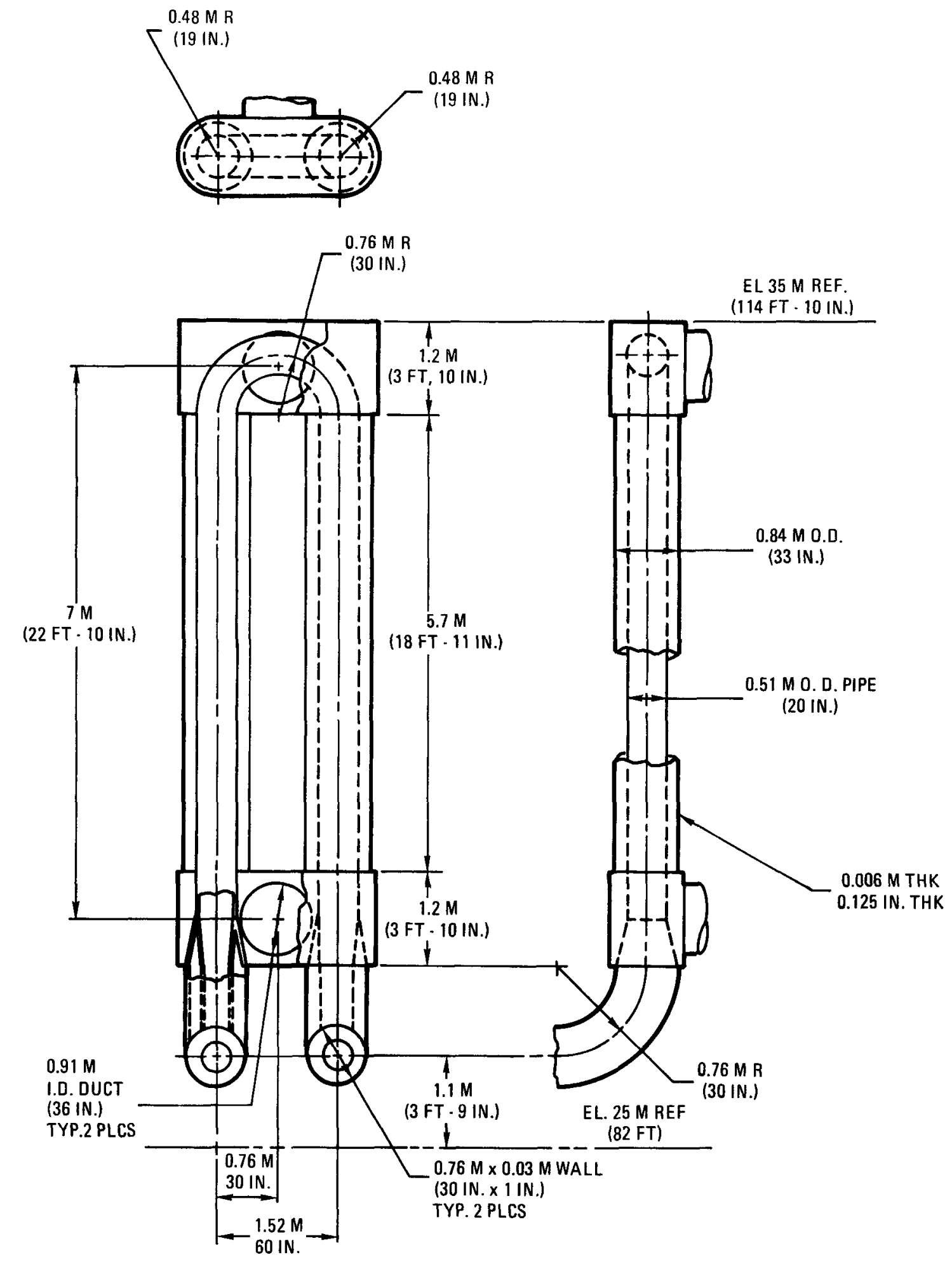

Fig. 5-6. Details of HHFTF heat exchanger 


\section{USE OF FACILITY FOR GASES OTHER THAN HELIUM}

So far the description of the HHFTF has been limited to helium. However, a unique feature of the facility will be its ability to operate with gases other than helium. First of all, the circulator bearing lubricant is water, which makes it compatible with any gas selected. The compressor Mach number at the inlet is less than 0.1 . If $\mathrm{CO}_{2}$ were used in the facility, the compressor inlet Mach number would be about 0.3. All other gases would have inlet Mach numbers ranging between helium and $\mathrm{CO}_{2}$.

For insulation qualities, all gases except hydrogen have five times the insulation capabilities of helium. Helium has a quite poor insulation quality but yields a relatively high heat conductivity value for a Kaowool-Saffil combination. Thus, the piping insulation will be more than adequate for all other gases. However, the low conductivity renders the heat exchanger less effective. The inner liner will have to be removed and the net power input to the gas will have to be limited to $40 \%$ of the input with helium. The costs for converting the HHFTF for utilizing other gases have been estimated, up to and including the flanged joints to which the component test loops could be coupled. These costs, which are preliminary, are given in Table 6-1. 
TABLE 6-1

SUMMARY OF $\operatorname{COSTS}(a)$ TO DESIGN, MANUFACTURE, AND COMMISSION THE HHFTF

\begin{tabular}{l|r}
\hline \multicolumn{1}{c|}{ Cost Item } & \multicolumn{1}{c}{ Cost } \\
\hline Architect-engineer & 200,000 \\
GA - helium piping design & 70,000 \\
GA - circulator design and analysis & 340,000 \\
GA - helium/air heat exchanger design & 60,000 \\
Rework existing vessel & 17,800 \\
New test vessel & 383,200 \\
Rework circulator & 313,700 \\
Piping and heat exchanger, construction and installation & 458,700 \\
Air blowers and silencer & 70,400 \\
Electrical rework & 53,500 \\
Data acquisition system & 101,800 \\
Equipment rental & 14,200 \\
Other services (gas, Dowtherm, oil, etc.) & 150,000 \\
Commissioning of facility & 360,000 \\
Allowance for indeterminates & $2,593,300$ \\
Total Cost & 518,660 \\
\hline
\end{tabular}

(a) January 1980 do11ars. 


\section{CONCLUSION}

It is recommended that the modifications proposed to the existing circulator test factility be made as soon as possible. This will allow the resulting hot helium flow test facility to be available to support all HTGR applications programs, whatever the future schedules may be.

In particular, the detail design of such a facility should start immediately. 